\title{
Estudio de la identidad cultural mediante una construcción epistémica del concepto identidad cultural regional
}

\author{
Study of cultural identity through an epistemic construction of the concept regional cultural \\ identity
}

Hugo Campos-Winter (hugo.campos@uach.cl) Universidad Austral de Chile (Valdivia, Chile) ORCID: 00000002-5039-9932

\begin{abstract}
The following article presents the formulation of the concept of regional cultural identity, based on a selection of the mental aspect of culture, which resulted in a narrative discursive definition. For this, metaphysical, linguistic and historical foundations are presented, and a contextualization composed of definitions of cultural identity, Latin American cultural identity and regional cultural identity. Regarding the latter, applications to the study of the Cultural Identity of the Region de los Rios are presented, due to the characteristics of this territory as a new region. The resulting concept describes the phenomenon as a sense of the regional being present in the cultural semiosphere, forming part of the collective memory and imaginary, manifested in the way of the thematic unity of a coherent and ordered historical narrative, progressively more complex and heterogeneous, which combines the linear progression of the subthemes and the constant theme of regional cultural identity.
\end{abstract}

Key words: identity, culture, Latin-American, national, regional.

\section{Resumen}

El siguiente artículo presenta la formulación del concepto identidad cultural regional, a partir de una selección del aspecto mental de la cultura, lo que dio como resultado una definición discursiva narrativa de identidad cultural regional. Para esto se presentan fundamentos metafísicos, lingüísticos e históricos, y una contextualización compuesta de definiciones de identidad cultural, identidad cultural latinoamericana e identidad cultural regional. Respecto de esta última, se presentan aplicaciones al estudio de la Identidad Cultural de la Región de los Ríos, debido a las características de este territorio como nueva región. El concepto resultante describe el fenómeno como un sentido del ser regional presente en la semiosfera cultural, formando parte de la memoria y del imaginario colectivo, manifestado en el modo de la unidad temática de una narración histórica coherente y ordenada, progresivamente más compleja y heterogénea, que combina la progresión lineal de los subtemas y el tema constante de la identidad cultural regional.

Palabras clave: identidad, cultural, latinoamericana, nacional, regional. 


\section{Introducción}

La identidad es un fenómeno de fundamental comprensión para las ciencias humanas. El diálogo sobre este fenómeno en la comunidad científica ha estado presente desde los inicios de las disciplinas. Actualmente es evidente su relevancia como fuente de sentido para la elevación del espíritu entendido como cultura. No obstante, la mayoría de los argumentos que tematizan la identidad están dirigidos a socavar o refutar las identidades individuales y del estado-nación. Esto en un contexto tecnocientífico y sociocultural de globalización que las ha problematizado como fuente de sentido tradicional. De acuerdo con Jaspers, este contexto refiere a que hoy en día "la existencia es hoy vista naturalmente como abastecimiento de masas en producción racional sobre la base de invenciones técnicas (...) aparato en marcha con el ritmo de una evolución económica concebida como necesaria" (Jaspers 1933:31). Este aparato en marcha que manifiesta la era de la técnica, implica la caída del espíritu, el cual habría alcanzado su más alto nivel en el tiempo-eje, a saber, "el presente no es un segundo tiempo-eje. Al revés, en el más agudo contraste con el primero, es una caída catastrófica en la indigencia en espíritu, humanidad, amor y fuerza creadora, donde solo una cosa, la producción científica y técnica, es sin duda, lo único grande en comparación con todo lo anterior" (Jaspers 1980:132).

Heidegger, en relación con los peligros de la esencia de la técnica moderna para la identidad del ser humano, refiere: "Pero cuando el peligro prevalece en el modo de la estructura de emplazamiento, entonces el peligro es supremo. Se nos muestra en dos perspectivas. Desde el momento en que lo no oculto aborda al hombre, no ya siquiera como objeto sino exclusivamente como existencias, y desde el momento en que el hombre, dentro de los límites de lo no objetual, es ya sólo el solicitador de existencias, entonces el hombre anda al borde de despeñarse, de precipitarse allí donde él mismo va a ser tomado sólo como existencia. Sin embargo, precisamente este hombre que está amenazado así se pavonea tomando la figura del señor de la tierra. Con ello se expande la apariencia de que todo cuanto sale al paso existe sólo en la medida en que es un artefacto del hombre. Esta apariencia hace madurar una última apariencia engañosa. Según ella parece como si el hombre, en todas partes, no se encontrara más que consigo mismo" (Heidegger 1994:14).

La total planificación técnica de la vida ha causado la vinculación transnacional de la economía, la sociedad y la cultura, lo que a su vez ha erosionado no solo la identidad del ser humano, sino también la identidad del estado-nación. Moulian refiere lo anterior de la siguiente forma, "la constante superación de las tecnologías, la destrucción de los parroquialismos, la erosión de los estrechos límites de los Estadosnacionales, la expansión obligada de la mirada desde nuestro ombligo hacia el mundo globalizado, implica un constante dinamismo" (Moulian 1997:46). Habermas, citando a Jaspers, refiere de que el Estado-nación como identidad unificadora ha terminado, "hoy día la idea del Estado-nación es una calamidad para Europa y para todos los continentes" (Habermas 1991:85).

A modo de síntesis de la problematización anterior, Lechner señala lo siguiente: "similar al choque de civilizaciones quinientos años atrás, el mapa cognitivo de las personas sufre un cambio radical (...) tiene lugar una redefinición de los límites espaciales (interno/externo) y se comprimen los horizontes temporales (antes/después), dejando a los individuos con pocos referentes simbólicos para situar su lugar en el mundo. La sociedad nacional sigue siendo el universo habitual de la vida cotidiana. Sin embargo, la experiencia de la gente ya no se agota en ese espacio. Las fronteras nacionales se difuminan, las distancias temporales se diluyen. Tanto el territorio de la nación como sus horizontes históricos pierden vigor" (Lechner 2002:104). 
Con todo, es un hecho de que en el contexto globalizado las identidades individuales y del estado-nación no se han perdido, pero si es necesario aceptar que se encuentran en un proceso de cambio. Para que este proceso tenga como resolución un fortalecimiento y proyección de tales identidades, es necesario valerse de otras identidades que no han formado parte importante de la discusión; las identidades culturales regionales. Desde estas, las identidades individuales y del estado-nación, obtienen y elaboran parte importante de su sentido. Esto porque es en las identidades culturales regionales donde se puede encontrar una gran riqueza de símbolos que componen la identidad cultural de los individuos. Y porque es a partir de la conjunción semiótica de las identidades regionales en un sistema interregional, que surge la identidad del estado-nación, la que, a su vez, recursivamente, otorga sentido a las primeras.

Clarificar el fenómeno de la identidad cultural regional es el objetivo de este artículo. Se indaga el aspecto mental o discursivo de la identidad cultural, puesto que este no ha sido suficientemente conceptualizado. Se realiza una construcción epistémica del concepto con base en fundamentos metafísicos, lingüísticos e históricos, y se presentan como contexto e insumos epistémicos las versiones de identidad cultural aplicadas a la identidad latinoamericana, nacional y regional. Respecto de esta última, se indaga la identidad cultural de la Región de los Ríos porque es un territorio con una reciente conformación administrativa como XIV Región, pero con un pasado cultural propio, lo que remite a una confluencia singular de relevancia investigativa.

En síntesis, este trabajo tiene por propósito llegar a una primera conceptualización discursiva de identidad cultural regional, que permita un análisis hermenéutico de los textos que constituyen la cultura mental de la Región de los Ríos.

\section{Metodología}

De acuerdo con Posner, la cultura se puede dividir en tres áreas de estudio, abordadas por tres subdisciplinas de la antropología respectivamente:

“a) El área de estudio de la antropología social es la cultura social, esto es, la sociedad. Cada sociedad consiste de instituciones y los rituales actuados por ellas. Los ejemplos incluyen instituciones religiosas, tales como una iglesia católica y los rituales de servicio de la Iglesia.

b) El área de estudio de la antropología material es la cultural material de una sociedad, esto es, su civilización. La civilización de una sociedad consiste de artefactos y habilidades de producirlos y usarlos. Ejemplos de artefactos usados en instituciones religiosas son cruces, hostias, rosarios, himnos, y biblias.

b) El área de estudio de la antropología cultural es la cultura mental de una sociedad, en la medida en que se manifiesta en su civilización, esto es, su mentalidad. La mentalidad de una sociedad consiste de artefactos mentales (esto es, las ideas y valores) y de convenciones que gobiernan sus usos y expresiones (...) Ejemplos de artefactos mentales religiosos son los santos católicos y sus emblemas, la clasificación de pecados con su correspondiente terminología ('pecado mortal', 'pecado venial', etc.), y los códigos gestuales de los sacerdotes" (Posner 2004:10).

Con base en esta clasificación, se seleccionó esta última categorización como el aspecto de la cultura a estudiar y como la más pertinente para obtener desde ella una definición discursiva de identidad cultural regional, por su localización en los discursos que tematizan lo regional. A su vez, se buscó lograr una nueva unidad discursiva referida al fenómeno identidad cultural regional, a partir de una revisión crítica de conceptualizaciones previas. 
A continuación, se presenta primero la síntesis conceptual obtenida y luego se exponen brevemente los fundamentos metafísicos, lingüísticos e históricos y la contextualización teórica del concepto.

\section{Síntesis conceptual}

A partir del análisis crítico realizado, se elaboró la siguiente conceptualización discursiva del fenómeno identidad cultural regional.

Es un sentido del ser regional presente en la semiosfera cultural, formando parte de la memoria como del imaginario colectivo. Se manifiesta en el modo de la unidad temática de una narración histórica coherente y ordenada, que combina la progresión lineal de los subtemas y el tema constante de la identidad cultural regional. Se compone de subtemas o subidentitades culturales adjetivadas como territoriales y supraregionales, interrelacionadas entre ellas antagónica o solidariamente, por incrustaciones descriptivas y retóricas.

Inicia con el relato de una situación de confluencia solidaria o antagónica de subidentidades en un tiempo y espacio determinado, haciendo emerger nuevas subidentidades que disputan la hegemonía de la unidad temática. Se desarrolla narrativamente como una serie progresiva de cambios de tipo coherente y ordenada, que se presenta en los estados individuales y relacionales de las subidentidades y de la hegemonía de la identidad regional. También pueden ocurrir cambios discontinuos causados por la inscripción, escamoteo o inserto de subidentidades. Tiende hacia estados de mayor diferenciación, pluralidad y heterogeneidad de las subidentidades en estados cada vez más integrados de la identidad regional.

\section{Fundamentos de la identidad cultural}

La identidad en general se manifiesta como mismicidad. Esto a partir de la afirmación de que el ente es ser y que el ente no es no ser; así se evidencia en el siguiente fragmento: "atención, pues; // que Yo seré quien hable: // Pon atención tú, por tu parte, en escuchar el mito: // cuáles serán las únicas sendas investigables del pensar. // Esta: // Del Ente es ser; del Ente no es no ser. Es senda de confianza, pues la verdad la sigue (...) Estotra: // Del Ente no es ser; y por necesidad, del Ente es no ser, // te he de decir que es senda impracticable y del todo insegura" (Parménides 1942:11).

En contraposición, la identidad se manifiesta también como relación internamente contradictoria de un ente, en otras palabras, como reflexión. Lo anterior se implica en los fragmentos de Heráclito 8, 88, 10: "enteros y no enteros, convergente y divergente, consonante y disonante: de todos uno y de uno todos (...) Una misma cosa es [en nosotros] lo viviente y lo muerto, lo despierto y lo dormido, y lo joven y lo viejo; éstos, pues, al cambiar, son aquéllos, y aquéllos, inversamente, al cambiar, son éstos (...) Lo que se opone es concorde, y de los discordantes [se forma] la más bella armonía, y todo se engendra por la discordia" (Mondolfo 2007:31).

Aristóteles se hace eco de la noción de identidad como mismicidad, y la establece como la lógica canónica del pensamiento occidental, el cual se proyecta hasta nuestros días, y que quedó sistematizada en la lógica ontológica con los principios de identidad y de no contradicción, y en la lógica proposicional del tercio excluido: 
$A=A$

A no es no $-\mathrm{A}$

No existe un tercer término que es a la vez A y no-A

En palabras del filósofo griego, el axioma de no contradicción/identidad es "el principio más firme de todos (...), a su vez, [es] aquel acerca del cual es imposible el error. Y tal principio es, necesariamente, el más conocido (...) y no es hipotético" (Aristóteles 2011:176). Aristóteles lo especifica con la siguiente proposición: "es imposible que lo mismo se dé y no se dé en lo mismo a la vez y en el mismo sentido" (Aristóteles 2011:177).

Con Hegel, el principio de identidad como mismicidad es puesto nuevamente en tensión. Según el filósofo alemán, la proposición de la identidad no es más que una ley del pensamiento abstracto que en su proposición $\mathrm{A}$ = A tiene ya su contradicción en sí misma, "puesto que una proposición aplaza también una diferencia entre sujeto y predicado, y ésta no realiza lo que su forma promete" (Hegel 2006:128). Un ente es $A=A$, a la vez y en el mismo sentido, luego $A$ es $-A$ en un segundo momento, $y$ en un tercer momento de síntesis $A$ que no es $n i+A$ ni $-A$, es $( \pm A)$. Por lo tanto, a la lógica de la identidad abstracta se le contrapone la lógica de la identidad como reflexión, donde la unidad internamente contradictoria es la síntesis y superación de las oposiciones. En otras palabras, lo que se define en Hegel es el método ontológico donde: “a) El pensamiento en cuanto entendimiento se queda parado en la determinidad fija y en la distintividad de ella frente a otra; un tal abstracto (así) delimitado vale para el entendimiento como siendo de suyo y subsistente. b) El momento dialéctico es el propio superar de tales determinaciones finitas y su pasar a sus opuestas. c) Lo especulativo racional-positivo aprehende la unidad de las determinaciones en su oposición, lo afirmativo que se contiene en la disolución de ellas y en su pasar" (Espinoza 2014:225).

Si la identidad es mismicidad o reflexión, es una pregunta que, para su respuesta, requiere preguntarse primero por su condición de posibilidad. Es decir, preguntarnos por el lenguaje. El lenguaje permite al ser humano concebir su identidad en el modo de la mismicidad o como reflexión. De esto se sigue que no hay una relación transparente del ser humano consigo mismo, puesto que este nace en un mundo objetivo ya donado de antemano. Este mundo objetivo es un continuum semiótico en el que el ser humano nace y habita junto a otros entes. Este continuum semiótico lo envuelve todo cuanto pueda ser pensado. Se encuentra entre y a través de los entes, y permite al ente que es el ser humano, concebirse como mismicidad o como reflexión. Esto es posible puesto que el ser humano es un ser simbólico.

Los símbolos están ya disponibles para el ser humano en el momento de su nacimiento. Le son donados a él por la comunidad semiótica de pertenencia. El lenguaje es el código simbólico más sofisticado, que presta su modo de semiosis a los otros códigos semióticos. El lenguaje es por tanto donado al ser humano. Con este, el ser humano encuentra las palabras para concebir su identidad en el modo de la mismicidad o de la reflexión. Las palabras son encontradas por el ser humano, porque estas ya están dispuestas en el continuun semiótico de la comunidad donde nace. Encontrando las palabras para conceptualizarse, dota de sentido su mundo propio y su ser, encontrando su identidad.

Por extensión, el lenguaje permite a una comunidad de seres humanos, conceptualizarse encontrando una identidad cultural. Digo encontrar una identidad, puesto de que el lenguaje se encuentra antes de cualquier comunidad determinada. Viene al encuentro de dicho ente. Es un don que el ser en general entrega a los seres humanos y que estos encuentran. El carácter ya disponible del lenguaje implica 
preguntarse por la facticidad de este ente. Su facticidad es que se encuentra compuesto de signos. Por lo tanto, comprender cómo el lenguaje permite conceptualizar nuestra identidad como mismicidad o como reflexión, implica preguntarse por la facticidad de los signos lingüísticos. Esto nos permitirá llegar a una definición de identidad cultural desde una perspectiva discursiva.

Saussure distingue entre identidad y diferencia, que evoca la distinción entre mismicidad y unidad internamente contradictoria, "el mecanismo lingüístico gira todo él sobre identidades y diferencias, siendo éstas las contrapartes de aquellas" (Saussure 1955:186). Para este autor, desde la perspectiva de la lingüística sincrónica, centrada en "las relaciones lógicas y psicológicas que unen términos coexistentes y que forman sistema, tal como aparecen a la conciencia colectiva" (Saussure 1955:174); la identidad sígnica (interna) es la correspondencia de los significantes y los significados en distintas instancias de habla en un mismo estado del sistema de la lengua. Pero también, la identidad del signo se muestra también como identidad (externa) dependiente de su diferenciación respecto de los signos solidarios previos y posteriores de la cadena sintagmática en un estado del sistema de la lengua (Saussure 1955:185).

Desde la perspectiva de la lingüística diacrónica, enfocada en "las relaciones que unen términos sucesivos no apercibidos por una misma conciencia colectiva, y que se reemplazan unos a otros sin formar sistema entre sí" (Saussure 1955:174); la identidad sígnica refiere a que entre dos signos aparentemente diferentes hay identidad porque se ha pasado de uno a otro mediante una serie de identidades sincrónicas en el habla sin que nunca el lazo que los une se haya roto por las transformaciones significantes sucesivas. Este lazo corresponde a lo que se mantiene en el nivel del significado o del concepto, del mismo modo en que a pesar de los cambios corporales de una persona y materiales de una comunidad, por ejemplo, el nombre propio sigue siendo el mismo, y lo sigue siendo no porque se componga del mismo significante ni porque mantenga sus relaciones con los mismos significantes diferenciales, sino porque a pesar de su separación de la cadena sintagmática que le es solidaria, se mantiene en relación sustitutiva con la cadena paradigmática virtual en la memoria individual y colectiva.

Mutatis mutandis, en Saussure se puede vislumbrar una puesta en práctica de los principios expuestos más arriba. La identidad sincrónica en su aspecto interno de mismicidad del signo en distintos momentos de habla en un estado de la lengua, es una aplicación de la identidad parmenidea-aristotélica. La identidad sincrónica en su aspecto externo pensada como efecto de las diferencias en un estado del sistema, es una aplicación en un nivel sistémico de la lógica heracliteana y del segundo momento de la lógica hegeliana. La identidad diacrónica es apropiada a la concepción de identidad como proceso dialéctico de Hegel. Por tanto, en la lógica interna del lenguaje, presentada por Saussure, vemos la identidad como mismicidad y como reflexión.

Si extendemos estas conclusiones a la identidad de un ser humano, el sentido del hombre como ser simbólico nos permite lo anterior, entonces tenemos que la identidad presenta tres aspectos. Primero, implica la mismicidad de una persona en distintas instancias de su trayectoria vital en un contexto más amplio que no cambia de estado, como puede ser la propia comunidad para el individuo. Segundo, implica una diferenciación de un ser humano respecto de su comunidad semiótica de origen, esto es, implica que la identidad resulta de la búsqueda del sí mismo mediante la imaginación que le permite un proyecto y libertad a cada existente. Tercero, implica lograr un proceso de integración y superación de las contradicciones que surgen internamente en un ser humano, manteniendo así recordatoriamente el sentido por sobre los cambios corporales. 
Respecto de una comunidad, implica mantener la mismicidad de esta frente a los cambios globales. Lo anterior se expresa en la tradición. En segundo lugar, implica lograr una diferenciación de la comunidad frente a su entorno global, evitando la colonización del sistema sobre el mundo de la vida cotidiana que caracteriza dicha comunidad, y lograr un destino común a partir de la apropiación del legado discursivo y la proyección de un destino común. En tercer lugar, implica que dicha comunidad logre superar sus contradicciones internas, manteniendo su sentido. Esto lo logra mediante el resguardo de la memoria colectiva.

Respondida la pregunta por el lenguaje, cabe preguntarse acerca de la temporización historiográfica de la identidad. Puesto el leguaje permite al ser humano encontrar su identidad, y aquél es un don del ser, pero el ser depende del tiempo, puesto que este es una condición de posibilidad no del ente, no de los existenciales del ser del Dasein, sino del ser en cuanto tal, en otras palabras, "el tiempo no puede estar presente en absoluto, no tiene en absoluto un determinado modo de ser, sino que él mismo es una condición de posibilidad de que haya tal cosa como el ser (no el ente)" (Heidegger 2004:321). Por ende, reflexionar sobre el tiempo es reflexionar sobre la verdad de la identidad. Pero la dificultad en agregar la dimensión temporal en la definición de identidad reside en la metafísica de la presencia de la civilización occidental. Respecto de esto, vemos una crítica radical en Heidegger a la metafísica de la presencia, proveniente desde Aristóteles.

Heidegger sostiene que el "ser del Dasein tiene su sentido en la temporeidad [es decir que desde aquello desde donde el Dasein comprende el ser, es el tiempo, y que la temporeidad es también] la condición que hace posible la historicidad como un modo de ser temporeo del Dasein mismo" (Heidegger 2015:45). Pero la temporeidad y la historicidad del Dasein, y por extensión de la historia universal, ha sido mal comprendida desde los inicios de la metafísica moderna, por la tradición filosófica de pensar el ser del ente como presencia, es decir, interpretado desde el tiempo presente. Frente a esto, Heidegger sostiene que el cuidado es el ser del Dasein, a saber, el "anticiparse-a-sí-estando-ya-en-(el-mundo) en-medio-de (el ente que comparece dentro del mundo)" (Heidegger 2015:216), y que este es esencialmente temporal. Esto puesto que son los éxtasis temporales los que dan sentido a los existenciales constitutivos del cuidado, a saber, "el anticiparse-a-sí (existencia) se funda en el futuro. El estar-ya-en (facticidad)... acusa en sí el haber-sido (pasado). El estar-en medio-de (caída)... es posible por la presentación (presente)" (Heidegger 2015:346).

Con otros Dasein, su destino comunitario estaría en el co-proyectarse, eligiendo las posibilidades heredadas por otros en el instante presente. De esto se desprende que la identidad cultural se manifiesta en la proyección de una comunidad apropiándose resolutivamente de su legado discursivo en el instante presente. Así, la identidad cultural pensada históricamente desde Heidegger queda constituida con una lógica de éxtasis temporales futuro-pasado-presente, donde es la capacidad de proyectarse la que da sentido a la memoria y a la acción en el instante presente. En sus palabras: "el peso esencial de la historia no recae ni en el pasado ni en el presente en su conexión con el pasado, sino en el acontecer propio de la existencia, que brota del futuro del Dasein" (Heidegger 2015:403).

\section{Versiones de identidad cultural}

Vergara del Solar, Vergara Estévez y Gundermann clasifican tres versiones de identidad cultural; la perspectiva esencialista, la historicista y la discursiva. La esencialista define la identidad cultural como una sustancia que es propia de un grupo humano y que no cambia a través del tiempo y del espacio. La versión 
historicista sostiene que la identidad cultural es algo abierto y en proceso de cambio por obra de la acción humana. Por último, la versión discursiva, define la identidad cultural como una construcción discursiva esencialmente narrativa. Aquí, desarrollaremos las dos últimas versiones, puesto que son insumo epistémico relevante para la construcción nuestro concepto.

Representante de la versión historicista en América Latina es Jorge Larraín, quien, con base en Stuart Hall, elabora una noción de identidad cultural referida a categorías sociales con las cuales los individuos se identifican, y así conforman un nosotros, a saber, "al construir sus identidades personales, los individuos comparten ciertas afiliaciones, características o lealtades grupales culturalmente determinadas, que contribuyen a especificar al sujeto y a su sentido de identidad. Implícita en esta afirmación está la idea de identidades colectivas tales como género, clase, etnia, religión, sexualidad, nacionalidad, etc., que Stuart Hall ha llamado 'identidades culturales'. Son formas colectivas de identidad porque se refieren a algunas características culturalmente definidas que son compartidas por muchos individuos" (Larraín 2014:35).

La identidad cultural se muestra en tales categorías sociales compartidas, formadoras también de las identidades personales, pero a la vez dependientes de su creación y recreación por parte de estas. Entre las identidades culturales, el autor nombra las identidades de clase, género, etnia, nación, entre las más importantes y universales.

También en la versión historicista, se observa la definición de Grínor Rojo, quien sostiene que la identidad cultural puede ser concebida en tres niveles, en palabras del autor, "propongo que subdividamos el concepto de identidad en tres categorías y/o niveles diferentes (...) dispuestas/os en un orden que se desplaza desde un grado de menor a uno de mayor abstracción, esas categorías y/o niveles son la/el de lo singular, la/el de lo particular y la/el de lo general o, si se quiere, aunque ello se preste a veces para regodeos metafísicos, la/el de lo universal" (Rojo 2006:29). De acuerdo con este autor, lo singular, refiere a los individuos como resultados únicos de la dialéctica interno/externo; lo particular, hace referencia a las identidades colectivas que coinciden con las identidades culturales de Jorge Larraín; y lo universal, hace referencia al ser humano con sus condiciones compartidas por todos, como por ejemplo el ser humano como ser simbólico.

La tercera versión postula que la identidad es una construcción discursiva, "una creación de carácter fundamentalmente narrativo o un conjunto de posiciones de sujeto (Foucault) entre las que nos movemos continuamente, desafiando las categorías rígidas con que intentamos dar cuenta de límites culturales que son continuamente traspasados" (Vergara del Solar et al. 2015:9).

Amalgamando versiones históricas y discursivas, estos autores desarrollan un concepto de identidad cultural como "un proceso de diferenciación de carácter intersubjetivo, nunca finalizado, siempre cambiante, mediado interactiva y comunicativamente, que permite el autorreconocimiento y la autonomía. Pero, el cual, también, está en la base de conflictos y formas de dominación entre Estados, naciones y grupos sociales, así como también, en otros casos, es un principio de resistencia frente a dicha dominación. Se construye desde la tradición (o mejor dicho desde sus interpretaciones) y, con frecuencia, en una relación crítica con ella. Las identidades culturales no se refieren únicamente al pasado, sino también al presente y al futuro, a lo que se quiere llegar a ser" (Vergara del Solar, Vergara Estévez y Gundermann 2010:62). 


\section{Identidad cultural latinoamericana, chilena y regional}

La identidad cultural regional, como hemos visto, es un fenómeno poco estudiado en comparación a la identidad individual y a la identidad nacional. De la identidad chilena, conviene hablar contextualizándola antes en la identidad latinoamericana. En efecto, la identidad latinoamericana y la identidad chilena se encuentran bien definidas en tipos ideales. Por lo demás, sirven para situar el metacontexto cultural latinoamericano y el contexto cultural chileno en que la identidad cultural de la Región de los Ríos se enmarca.

Los autores Vergara del Solar y Vergara Estévez, con base en su definición de identidad cultural ya expuesta, presentan una síntesis de las investigaciones donde se observan tres versiones de identidad cultural latinoamericana, la versión indigenista, desde donde se afirma una paradoja, ya "que dicha identidad no es 'latinoamericana' sino indígena, puesto que nuestra región nunca ha dejada de ser indígena en sus aspectos esenciales. La cultura europea no habría sido entre nosotros más que una yuxtaposición colonial o neocolonial, una cultura imaginaria o una apariencia de modernidad" (Vergara del Solar y Vergara Estévez 2005:33), por lo tanto, Latinoamérica tendría una esencia indígena, que se encarna en reivindicaciones y producción de saber indianista.

La versión hispanista sostiene que "somos hispanos o herederos de dicha cultura (...) [que] los indios habrían estado viviendo en estado de naturaleza (...) [y que] la conquista se interpreta como una conquista espiritual de evangelización y extensión de la civilización occidental" (Vergara del Solar y Vergara Estévez 2005:35).

La versión occidentalista se opone a las anteriores y plantea que "somos occidentales o podríamos llegar a serlo, a diferencia de Asia y África, América Latina no presentaría culturas tradicionales vigentes, completamente distintas a la occidental" (Vergara del Solar y Vergara Estévez 2005:36).

Por último, la versión del mestizaje cultural o latinoamericanista, desde donde se afirma que "existen una identidad y una cultura latinoamericanas. Ellas serían el resultado de una síntesis de distintos elementos culturales provenientes de las sociedades amerindias, europeas y africanas" (Vergara del Solar y Vergara Estévez 2005:37), las cuales en su conjunción producirían una síntesis cultural mestiza que implica una forma particular de ser en el mundo representado en parte por el barroco latinoamericano.

Con respecto a la identidad del estado-nación chileno, Larraín elabora las siguientes versiones de identidad presentes en la historia de la nación, dividiéndolas en versiones específicas y versiones tradicionales según sean propiamente chilenas o compartidas con el resto de los países latinoamericanos. Con respecto a las primeras, se presenta la versión esencialista militar-racial que se ha construido sobre la base de diferentes fuentes, las cuales confluyen en la "insistencia del rol de los elementos bélicos y militares en la identidad chilena" (Larraín 2014:145). La identidad chilena, desde esta versión, se ha forjado en guerras victoriosas, por el rol central del ejército en la formación del Estado y la nación chilena, y por ser encarnada por el roto chileno, resultado de la amalgama entre el godo germano conquistador y la raza araucana guerrera.

La versión psicosocial, fundada en "varios estudios que se caracterizan por la búsqueda de los rasgos psicosociales del chileno y que pretenden configurar lo que sería "el carácter chileno»" (Larraín 2014:155), es resultado de la sumatoria de los caracteres particulares, como por ejemplo la voluntad de ser y la aspiración al orden. 
La versión empresarial, la cual comenzó a construirse durante la década de los noventa, donde prevalecieron sin contrapeso las políticas neoliberales, y que han tenido como correlato las políticas de identidad en el ámbito cultural. En este tiempo se empezó a considerar a Chile como "un país emprendedor" (Larraín 2014:159). Desde esta versión de identidad, nos vemos a nosotros mismos como un país con pretensiones de desarrollo y con una cultura consumista.

Finalmente, la versión de la cultura popular, "discurso claramente no dominante pero con características distintivas, que destaca como lo más propio de la identidad chilena la cultura popular" (Larraín 2014:168). Esta identidad es representada en el pueblo y su imaginación, creatividad y autonomía frente a las dificultades.

Con respecto a las versiones tradicionales, se muestra la versión esencialista hispanista, "la cual es una de las más elaboradas y sistemáticas con respecto a los problemas de identidad latinoamericana y chilena" (Larraín 2014:175). Deriva de la conquista y se expresa en una sangre española a partir de la cual tenemos un espíritu creativo y realista, y vivimos en un imaginario donde los actores principales son Dios, el Rey y el Pueblo.

También tenemos la versión esencialista religiosa, la cual se formó "en el encuentro entre los valores culturales indígenas y la religión católica traída por los españoles” (Larraín 2014:181), a partir de lo cual, tenemos un sustrato católico o al menos cristiano y una cultura barroca de carácter oral y racionalidad sapiencial alienada por las elites y expresada en la religión popular.

Desde Boisier, la identidad cultural regional sería una construcción social clave del desarrollo del sistema regional, ya que esta permitiría además de la generación de una red de actores e instituciones, la confluencia de los recursos económicos en corrientes de desarrollo regional, cumpliendo con una función de activación del desarrollo. En este sentido, "el desarrollo cultural e identitario debe ser un generador de valores estéticos y de sentido de pertenencia regional y promotor del desarrollo socio-económico y de la innovación" (Gobierno Regional de los Ríos 2010:121).

La versión de identidad como fuente de desarrollo de la región, la vemos también de forma similar en Amtmann, en quien se agrega un notable énfasis en el compromiso afectivo con la historia de la región, a partir del cual puede construirse un proyecto común que subsuma la conflictividad intrínseca a esta categoría, que ya veíamos en Vergara y Gundermann. Así, para el autor la identidad cultural regional "implica un compromiso afectivo vital con el pasado, presente y futuro de los procesos económicossociales y culturales que acaecen en una localidad o región. Este compromiso vital, entonces, es una fuerza social para asumir el proyecto de desarrollo compartido por los actores, al que pueden subsumirse los intereses conflictivos entre categorías de actores" (Amtmann 1997:9).

Cabe destacar que el autor pone en valor las acciones comunicativas y conversacionales más específicas como fuentes de generación de identidad cultural regional. Además, se observan en la definición dos nuevos aspectos de la identidad cultural, el descubrimiento y el compromiso vital. En el mismo sentido de vinculación identidad-desarrollo regional, Fortoul sostiene la siguiente definición de identidad cultural regional: "la diversidad es combustible de vitalidad y creatividad para el desarrollo. Su fuente nutricia es la identidad [regional], no como patrimonio codificado sino como permanente invención de la sociedad que procesa sus tendencias internas y recibe y asimila las influencias externas. Las combina, las recrea y las transforma, no como actitud contemplativa hacia un pasado estático, de grandes o pequeñas 
tradiciones, sino como una síntesis animada, inimitable y jamás concluida. En estos términos, la identidad es condición de progreso de personas, grupos y sociedades" (Fortoul 2003:12).

Una última versión de identidad cultural regional, es elaborada por Vergara del Solar y Gundermann. Para los autores, la identidad regional es conceptualizada como una identidad cultural en el sentido de ser, de acuerdo con Bourdieu, un campo regional, en nuestra perspectiva, la identidad regional constituiría lo que, siguiendo a Bourdieu, puede denominarse 'campo regional', el que está conformado por distintas identidades culturales o subidentidades. Estas subidentidades individuales y colectivas eventualmente entran en conflicto por la jerarquía de lealtades entre ellas y por la apropiación simbólica de lo regional, en el sentido gramsciano de hegemonía cultural en tanto capacidad de liderazgo moral e intelectual. Asimismo, las identidades culturales que componen la dinámica interna de la identidad regional pueden ordenarse en dos ejes; 'uno territorial, que puede ordenarse en niveles 'inferiores' y 'superiores' de acuerdo a su mayor o menor amplitud espacial; y un eje horizontal, correspondiente a las identidades culturales no territoriales o transregionales" (Vergara del Solar y Gundermann 2012:119).

En el primer eje vertical se ordenan en niveles inferiores y superiores de acuerdo con su amplitud espacial, donde los autores encuentran cinco espacios diferenciados de apropiación identitaria del espacio: barrio, localidad o pueblo, ciudad, área y región; en el segundo eje horizontal o supraregional se encuentran las identidades que trascienden el espacio regional y son compartidas por otras regiones y a nivel nacional. Los autores destacan que la identidad cultural regional no coincidiría punto a punto con la delimitación política administrativa, porque esta muchas veces no considera en su magnitud la historia cultural de la comunidad regional concreta y también por factores actuales de migración, desplazamientos internos, intercambio cultural y la internacionalización de la cultura de masas. Por lo que se debe considerar de forma provisoria la delimitación política administrativa y ver si esta coincide con la presencia de identidades culturales que participen del imaginario regional y sean reconocidas como tales. Por lo anterior, para los autores, basados en la noción de complejidad de Niklas Luhmann, la identidad cultural regional posee una dinámica compleja en el sentido de que se debe realizar una selección entre sus elementos para funcionar cada vez, de tal modo que "no todas las identidades ni todas las relaciones entre ellas pueden activarse simultáneamente. En determinados momentos y circunstancias, algunas aparecerán en primer plano, mientras las demás quedarán en un estado latente dentro del horizonte de posibilidad del sistema. Lo mismo puede decirse de las relaciones entre ellas" (Vergara del Solar y Gundermann 2012:120).

A partir de esta distinción, Vergara del Solar y Gundermann entregan una visión de la dinámica interna de la identidad cultural regional de la Región de los Lagos en comparación con la Región de Tarapacá. Aquí presentaremos algunos elementos culturales de la primera región nombrada, habida cuenta de que desde esta emergió la Región de los Ríos.

La provincia de Valdivia parece ser la identidad local históricamente más relevante, seguida por Osorno y Llanquihue. En el imaginario de esta provincia la historia colonial ha tenido menos importancia que la diferenciación con respecto a la identidad nacional, destacándose la propuesta federalista. La identidad germana y germana-chilena destacan por sus aportes a la economía y a la cultura, y la identidad mapuche se presenta más bien como una identidad subalterna dentro del campo regional. Entre mapuches y germano-chilenos han existido relaciones pacíficas de comercio y trabajo sustituidas intermitentemente por antagonismos con conflictos latentes por el dominio de las tierras, especialmente en épocas de 
gobiernos populares, pero que no han pasado a convertirse en conflictos patentes en la región, imperando la relación pacífica.

Finalmente, con respecto a investigaciones previas sobre la identidad cultural de la Región de los Ríos, propuse una versión desde una posición interdisciplinaria y desde un enfoque discursivo y construccionista, entendiendo en primer lugar la identidad como posicionamiento, término obtenido desde Bronwyn y Harré, referente a "un proceso discursivo donde las identidades se localizan en conversaciones en las que participantes, observable y subjetivamente coherentes, producen argumentos" (Campos 2016:87). En este sentido "el sujeto (y su identidad) es la serie o conglomerado de posiciones, de posiciones de sujeto, provisionales (explícitas o implícitas), y no necesariamente indesarmables, en que una persona es momentáneamente puesta por los discursos y el mundo donde habita" (Campos 2016:87). Esto, se ensambló con la definición semiótica de cultura obtenida desde Clifford Geertz, a saber, "el concepto de cultural que propugno y cuya utilidad procuran demostrar los ensayos que siguen es esencialmente un concepto semiótico. Creyendo con Max Weber que el hombre es un animal inserto en tramas de significación que él mismo ha tejido, considero que la cultura es esa urdimbre y que el análisis de la cultura ha de ser, por lo tanto, no una ciencia experimental en busca de leyes, sino una ciencia interpretativa en busca de significaciones" (Geertz 2003:20). Lo cual dio como resultado una definición de identidad cultural regional como la serie de posicionamientos presentes en el discurso sobre la región. Lo anterior con la premisa de que ambas nociones de identidad (individual y cultural) tienen como materia constituyente el discurso, así entonces, desde esta perspectiva "la identidad individual [es] a la serie de posicionamientos individuales, lo que la identidad cultural regional [es] a la serie de posicionamientos presentes en el discurso sobre la Región de los Ríos" (Campos 2016:88).

A partir de esta definición de identidad cultural regional y de su aplicación en el análisis de la Política Cultural Regional de la Región de los Ríos 2011-2016, se obtuvo una identidad cultural de la Región constituida por un conglomerado de identidades culturales divididas en reforzadas o socavadas según la política cultural argumentara a favor o en contra de estas. Las identidades reforzadas fueron: la política cultural como sujeto constituyente y como expresión de un gobierno benefactor; españoles generadores de la historia regional y fuentes del conocimiento histórico; alemanes intelectuales e industriosos, franceses emprendedores, Iglesia Católica como guardiana de nuestro conocimiento histórico y Universidad Austral de Chile como protagonista cultural. Entre las identidades socavadas se encuentran: ciudad de Valdivia y la Región con una historia heterónoma, administración local desordenada y cultura decadente, y mapuches relativizados en su existencia histórica.

Vemos cómo en las dos últimas definiciones de identidad cultural regional aplicadas a la Región de los Ríos, una desde la sociología otra desde la psicología, hay coincidencias en concebir la identidad cultural como una totalidad internamente diferenciada, campo en la primera, conglomerado en la segunda. A esta última se le agrega el modo de la serie. Por su parte, la primera definición considera explícitamente la condición de diferenciación, conflictividad e historicidad de la identidad regional, cualidades que no son observadas en la definición propia. Finalmente, una diferencia importante para la conceptualización es que en la primera definición se da mayor énfasis a un análisis del discurso como representación mientras que la segunda se enfoca en el discurso como acción con eventuales efectos semántico-pragmáticos. 


\section{Conclusión}

Como se hecho ver la identidad cultural es un fenómeno complejo, conceptualizado desde múltiples perspectivas que le otorgan una diversidad de aspectos. No obstante, al menos las definiciones analizadas confluyen y se complementan en algunos puntos en común. En primer lugar, todas pueden ser ubicadas dentro de la categorización primaria identidad como mismicidad o identidad como reflexión. El análisis lingüístico permite una integración de tal dicotomía desde una perspectiva discursiva narrativa. Asimismo, la temporización histórica del concepto es una condición de posibilidad fundamental que permite encontrar la verdad sobre la identidad cultural. Además de sus fundamentos, la identidad cultural puede ser abordada desde distintos niveles, por ejemplo, nacional, regional, urbano, barrial, individual, etc. También, puede ser analizada desde sus múltiples tipos ideales, a saber, clase, género, sexo, nacionalidad, rango etario, etc. A este concepto se le atribuyen aspectos semióticos, sociales y materiales, además de estar atravesado por relaciones de poder, conflicto y solidaridad. Por último, las identidades culturales tienen efectos semántico-pragmáticos en los territorios y/o colectivos e individuos que se identifican con dichas identidades.

Por su parte, la identidad cultural regional tiene valor en sí misma para la elevación del espíritu de los territorios regionales, pero también como recurso para fortalecer las identidades culturales individuales y del estado-nación. No obstante, como se vio, la identidad cultural regional en su aspecto mental no está suficientemente clarificada. Por lo anterior, se desarrollaron fundamentos metafísicos, lingüísticos e históricos para construir el concepto discursivo de identidad cultural regional. Asimismo, se presentaron y analizaron versiones de identidad cultural, identidad cultural latinoamericana, identidad cultural chilena, identidad cultural regional, y aplicaciones de esta última a la Región de los Ríos. Estas sirvieron de contexto e insumo epistémico para desarrollar el concepto discursivo de identidad cultural regional. Este aborda en una dimensión sincrónica la identidad cultural regional como un sistema constituido por la dinámica hegemónica de las subidentidades culturales que componen la identidad cultural regional. Asimismo, en una dimensión diacrónica, aborda el progreso no sistemático de las subidentitades culturales. La identidad cultural regional, tal y como se ha definido, tiende progresivamente o por saltos hacia mayores grados de pluralidad, diferenciación y heterogeneidades de las subidentitades que la componen, y hacia un mayor grado de integración de las mismas en el sistema identitario pensado como totalidad compuesta de una dinámica hegemónica.

\section{Bibliografía}

Aristóteles. 2011. Metafísica. Madrid: Gredos.

Amtmann, C. 1997. Identidad regional y articulación de los actores sociales en procesos de desarrollo regional. Revista Austral de Ciencias Sociales 1: 5-14. http://mingaonline.uach.cl/pdf/racs/n1/art01.pdf

Campos, H. 2016. Política cultural y construcción de identidad regional. CUHSO Cultura-Hombre-Sociedad 26(1): 81-108. https://doi.org/10.7770/cuhso-V26N1-art1012

Espinoza, R. 2014. Hegel y la filosofía de la complejidad: el método por excelencia, pp. 211-229. En: E. Bustos, P. Marquet y A. Palacios (Eds.) Las rutas de la complejidad. Valparaíso: Ediciones Instituto de Sistemas Complejos de Valparaíso.

Fortoul, F. 2003. Identidad y desarrollo. Revista Austral de Ciencias Sociales 7: 5-16. http://mingaonline.uach.cl/pdf/racs/n7/art02.pdf 
Geertz, C. 2003. La interpretación de las culturas. Barcelona: Gedisa.

Gobierno Regional de los Ríos. 2010. Estrategia de desarrollo regional. Osorno: GORE de los Ríos. https://www.opia.cl/static/website/601/articles-77312_archivo_02.pdf

Habermas, J. 1991. Yet again: German Identity: A unified nation of angry DM-Burghers? New German Critique 52: 84-101. https://doi.org/10.2307/488190

Hegel, G. W. F. 2006. Filosofía de la lógica. Buenos Aires: Editorial Claridad.

Heidegger, M. 2015. Ser y tiempo. Santiago: Editorial Universitaria.

Heidegger, M. 2004. Lógica. La pregunta por la verdad. Madrid: Alianza editorial.

Heidegger, M. 1994. Conferencias y artículos. Barcelona: Ediciones del Serbal.

Jaspers, K. 1980. Origen y meta de la historia. Madrid: Alianza Editorial.

Jaspers, K. 1933. Ambiente espiritual de nuestro tiempo. Barcelona: Editorial Labor.

Larraín, J. 2014. Identidad chilena. Santiago: LOM.

Lechner, N. 2002. Las sombras del mañana. La dimensión subjetiva de la política. Buenos Aires: EUDEBA.

Mondolfo, R. 2007. Heráclito. Textos y problemas de su interpretación. México: Siglo XXI editores.

Moulián, T. 1997. Chile actual. Anatomía de un mito. Santiago: Arcis-LOM.

Parménides. 1942. El poema de Parménides. México: Imprenta Universitaria.

Posner, R. 2004. Basic tasks of cultural semiotics, pp.56-89. En: G. Withalm \& J. Wallmannsberger (Eds.). Signs of power - power of signs. Essays in Honor of Jeff Bernard. Vienna: INST.

Rojo, G. 2006. Globalización e identidades nacionales y postnacionales... ¿de qué estamos hablando? Santiago: LOM.

Saussure, F. 1955. Curso de lingüística general. Buenos Aires: Editorial Losada

Vergara del Solar, J. y Gundermann, H. 2012. Conformación y dinámica interna del campo identitario regional en Tarapacá y Los Lagos, Chile. Chungara 44(1): 115-134. https://doi.org/10.4067/S071773562012000100009

Vergara del Solar, J. y Vergara Estévez, J. 2005. Los dilemas de la identidad. Una mirada sociológica acerca de la identidad cultural latinoamericana. Estudios Latinoamericanos 32(40): 27-48.

http://www.revistas.unam.mx/index.php/rel/article/view/50363

Vergara del Solar, J; Vergara Estévez, J. y Gundermann, H. 2015. Sociología e identidad cultural latinoamericana. Revista de Estudios Cotidianos 1(3): 1-33.

https://dialnet.unirioja.es/servlet/articulo?codigo $=5155234$

Vergara del Solar, J; Vergara Estévez, J. y Gundermann, H. 2010. Elementos para una teoría crítica de las identidades culturales en América Latina. Utopía y Praxis Latinoamericana 15(51): 57-79.

http://www.redalyc.org/articulo.oa?id=27916299005

Recibido el 20 Feb 2018

Aceptado el 14 May 2018 